\title{
Le concours de praticien hospitalier en médecine d'urgence
}

\section{The entrance exam of hospital practitioner in emergency medicine}

\author{
D. Pateron \\ (C) SFMU et Springer-Verlag France 2013
}

Le passage du concours de praticien hospitalier en médecine d'urgence est un moment important de la vie professionnelle des urgentistes. Il permet d'être inscrit sur la liste d'aptitude et de postuler comme praticien hospitalier dans les établissements publics de santé. Cette démarche, qui s'inscrit dans le parcours professionnel de l'urgentiste, l'engage dans la durée et l'appelle à participer à l'organisation pérenne des structures d'urgence de l'hôpital public. L'article « Le concours de praticien hospitalier en médecine d'urgence : mode d'emploi », rédigé par les responsables des principales composantes de la médecine d'urgence et coordonné par Luc Marie Joly, est un document très instructif pour l'ensemble des futurs candidats [1]. Il faut saluer le caractère pédagogique et la transparence de ce document. Les membres du jury 2012 y retrouvent une très grande partie de leurs réflexions et leur vécu du concours de cette année.

L'implication des acteurs de terrain de la médecine d'urgence dans la constitution des jurys de ce concours spécifique, récemment créé, témoigne de la volonté du Centre national de Gestion de reconnaître notre activité comme une spécialité à part entière.

L'ensemble des aspects relatifs au déroulement du concours sont abordés dans 1'article. Quelques points particuliers, mais au combien important, méritent d'être soulignés :

- les grilles de notation sont un élément très important pris en compte par le jury. Elles doivent guider les candidats dans la constitution de leur dossier. Le jury reste toutefois souverain et libre de les modifier d'une année à l'autre ;
- les conseils prodigués en fin d'article résument sans équivoque le constat réalisé par le jury 2012 ;

- les jeunes praticiens récemment diplômés sont invités à acquérir une expérience conséquente et à moins se précipiter pour se présenter au concours. La qualité évidente de certains candidats pâtit indiscutablement d'un manque d'acquis d'expérience ;

- l'implication dans la vie et le fonctionnement de la structure d'urgence dans laquelle évolue le candidat est un élément important de l'appréciation générale par les membres du jury ;

- la connaissance du monde de la médecine d'urgence et l'implication du candidat dans ses structures, autant nationales que régionales, sont également des éléments de poids.

Comme l'indiquent les auteurs de l'article qui fait l'objet de cet éditorial, le concours de praticien hospitalier de médecine d'urgence n'est en aucune manière une formalité. Ainsi, la réussite des candidats à cette épreuve impose une implication forte des aînés dans la préparation du concours présenté par les plus jeunes qui doivent savoir faire preuve de patience. Ces quelques conseils prodigués invitent aussi les « trop » jeunes candidats à ne pas se décourager en cas d'un premier échec.

\section{Référence}

1. Joly LM, Carli P, Ducassé JL, et al (2013) Le concours de praticien hospitalier en médecine d'urgence : mode d'emploi. Ann Fr Med Urgence 3:193-9

\footnotetext{
D. Pateron $(\bowtie)$

Service des urgences, hôpital Saint-Antoine, Assistance publique-hôpitaux de Paris (AP-HP), 184, rue du Faubourg Saint antoine, F-75012 Paris, France e-mail : dominique.pateron@sat.aphp.fr
}

Université Pierre et Marie Curie-Paris VI, Paris, France

Président du Jury du Concours de praticien hospitalier en médecine d'urgence 2012 